\title{
Comparative Diagnostic Accuracy of Ganglion Cell-Inner Plexiform and Retinal Nerve Fiber Layer Thickness Measures by Cirrus and Spectralis Optical Coherence Tomography in Relapsing-Remitting Multiple Sclerosis
}

\author{
Julio J. González-López, ${ }^{1,2,3}$ Gema Rebolleda, ${ }^{1,2}$ Marina Leal, ${ }^{1}$ Noelia Oblanca, ${ }^{1}$ \\ Francisco J. Muñoz-Negrete, ${ }^{1,2}$ Lucienne Costa-Frossard, ${ }^{4}$ and José C. Álvarez-Cermeño ${ }^{4}$ \\ ${ }^{1}$ Department of Ophthalmology, Ramón y Cajal University Hospital, Carretera de Colmenar Km 9, 1, 28034 Madrid, Spain \\ ${ }^{2}$ Department of Surgery, Alcalá de Henares University, Madrid, Spain \\ ${ }^{3}$ Medical Retina Department, Moorfields Eye Hospital, London, UK \\ ${ }^{4}$ Department of Neurology, Ramón y Cajal University Hospital, Madrid, Spain
}

Correspondence should be addressed to Gema Rebolleda; grebolleda@telefonica.net

Received 29 July 2014; Revised 23 August 2014; Accepted 26 August 2014; Published 14 September 2014

Academic Editor: Antonio Ferreras

Copyright (C) 2014 Julio J. González-López et al. This is an open access article distributed under the Creative Commons Attribution License, which permits unrestricted use, distribution, and reproduction in any medium, provided the original work is properly cited.

\begin{abstract}
Objective. To estimate sensitivity and specificity of several optical coherence tomography (OCT) measurements for detecting retinal thickness changes in patients with relapsing-remitting multiple sclerosis (RRMS), such as macular ganglion cell-inner plexiform layer (GCIPL) thickness measured with Cirrus (OCT) and peripapillary retinal nerve fiber layer (pRNFL) thickness measured with Cirrus and Spectralis OCT. Methods. Seventy patients (140 eyes) with RRMS and seventy matched healthy subjects underwent pRNFL and GCIPL thickness analysis using Cirrus OCT and pRNFL using Spectralis OCT. A prospective, crosssectional evaluation of sensitivities and specificities was performed using latent class analysis due to the absence of a gold standard. Results. GCIPL measures had higher sensitivity and specificity than temporal pRNFL measures obtained with both OCT devices. Average GCIPL thickness was significantly more sensitive than temporal pRNFL by Cirrus ( $96.34 \%$ versus $58.41 \%)$ and minimum GCIPL thickness was significantly more sensitive than temporal pRNFL by Spectralis (96.41\% versus $69.69 \%)$. Generalised estimating equation analysis revealed that age $(P=0.030)$, optic neuritis antecedent $(P=0.001)$, and disease duration $(P=0.002)$ were significantly associated with abnormal results in average GCIPL thickness. Conclusion. Average and minimum GCIPL measurements had significantly better sensitivity to detect retinal thickness changes in RRMS than temporal pRNFL thickness measured by Cirrus and Spectralis OCT, respectively.
\end{abstract}

\section{Introduction}

Relapsing-remitting multiple sclerosis (RRMS) is a chronic, immune-mediated demyelinating disease of the central nervous system that frequently involves the visual pathways, usually in the form of optic neuritis (ON) [1]. Postmortem analysis revealed optic nerve lesions in $94-99 \%$ of RRMS patients, even in the absence of a clinical history of ON [2].

Optical coherence tomography (OCT) is a noninvasive and reproducible tool for evaluating the retinal and optic disc anatomy of patients with this clinical disorder. It uses low-coherence interferometry to obtain detailed images of the retinal architecture. Modern high-speed spectral-domain (SD) OCT devices can obtain high resolution images of the retina. Computerised algorithms can be used on these images in order to automatically identify and obtain thickness measurements of discrete retinal layers, including the retinal nerve fiber layer (RNFL) and the macular ganglion cell-inner plexiform layers (GCIPL) [3].

In patients with RRMS, the main focus has been the evaluation of the peripapillary retinal nerve fiber layer (pRNFL). The RNFL contains the unmyelinated axons originating from 
the ganglion cell neurons. In a previous report, we found that Spectralis showed a significantly higher thinning for temporal quadrant than Cirrus in eyes of RRMS patients, suggesting that $\mathrm{N}$-site axonal analysis could define axonal damage in relapsing-remitting multiple sclerosis patients earlier than conventional pRNFL analysis [4].

Optic nerve demyelination, due to clinical or subclinical $\mathrm{ON}$, can result in retrograde degeneration of the optic nerve axons, leading to RNFL and GCIPL thinning $[5,6]$. In fact, several studies have reported statistically significant thinning of the pRNFL and GCIPL in patients with RRMS with and without optic neuritis compared to healthy control subjects [7-10].

Moreover, macular GCIPL thickness has been found to have better structure-function correlations than pRNFL thickness with both visual function and disability in RRMS patients [11]. At least in part, this observation may be due to the superior reproducibility of GCIPL over pRNFL thickness measurements [11].

Thus, we hypothesize that GCIPL measurements can be more sensitive to and specific of retinal involvement in patients with RRMS than those with pRNFL measurements.

The main purpose of this study was to estimate the sensitivity and specificity of macular GCIPL thickness measured with the Cirrus OCT ganglion cell analysis (GCA) algorithm (Carl Zeiss Meditec AG, Jena, Germany) and pRNFL thickness analysis with Cirrus and Spectralis (Heidelberg Engineering GmbH, Heidelberg, Germany) OCTs in detecting retinal thickness changes in eyes from patients with a clinical diagnosis of RRMS versus age-matched normal subjects using latent class analysis.

Ancillary objectives were to quantify color-code abnormalities in GCIPL and pRNFL measures by Cirrus and Spectralis OCTs in eyes from patients with RRMS and healthy control subjects, to quantify the correlations of GCIPL and pRNFL measurements with the visual function and disability in MS patients, and to study the effect of optic neuritis antecedent on the obtained measurements.

\section{Methods}

2.1. Subjects. An observational, prospectively recruited, cross-sectional study was performed. The study was approved by the Research Ethics Committee in the Ramón y Cajal University Hospital. All research complied with the tenets of the declaration of Helsinki, and all subjects participating in the study gave their written informed consent. Confidentiality of participating subjects was protected throughout the study.

We included 70 patients with a diagnosis of RRMS and 70 healthy control subjects. Patients were enrolled consecutively from the Neuroophthalmology Department from January 2012 to September 2012. Healthy controls without a history of neurological and ophthalmological disease were recruited among the hospital staff.

Diagnosis of RRMS was based on McDonald criteria by the treating neurologist [12]. None of the included patients had a diagnosis of secondary progressive multiple sclerosis.
All participants underwent a complete neuroophthalmic evaluation that included pupillary, anterior segment, and funduscopic examinations; assessment of logMAR best corrected visual acuity (BCVA), and they were scanned after pupillary dilation with Cirrus (Carl Zeiss Meditec AG, Jena, Germany) and Spectralis (Heidelberg Engineering $\mathrm{GmbH}$, Heidelberg, Germany) OCTs on the same day in random order. Both eyes of each subject were included. Exclusion criteria were intraocular pressure of $21 \mathrm{~mm} \mathrm{Hg}$ or higher, an optic disc suspicious for glaucoma under dilated funduscopy, a refractive error greater than 5.0 diopters (D) of spherical equivalent or $3.0 \mathrm{D}$ of astigmatism in either eye, media opacity, a recent history of optic neuritis 6 months prior to the day of imaging, systemic conditions that could affect the visual system, a history of ocular trauma, or concomitant ocular diseases, including glaucoma.

Related medical records were carefully reviewed, including the duration of the disease, the Expanded Disability Status Scale (EDSS) scored by a neurologist (LC), and the presence of prior episodes of optic neuritis (ON) as reported by the treating neurologist and the patient.

The visual field (VF) was tested only in the eyes of patients with RRMS, using a Humphrey Field Analyzer (Carl Zeiss Meditec AG, Jena, Germany) and the SITA Standard protocol (program 24-2). VF test was considered reliable when fixation losses were less than $20 \%$ and false-positive and false-negative errors were less than $15 \%$.

2.2. Optical Coherence Tomography Measurements. A single, well-trained optometrist (NO) performed all OCT examinations in random order to prevent any fatigue bias. All poor-quality scans were rejected, defined as those with signal strength of $\leq 6$ by Cirrus. For Spectralis OCT only those images with a signal-to-noise score higher than $25 \mathrm{~dB}$ were analyzed. Scans with misalignment, segmentation failure, decentration of the measurement circle, and poor illumination or those out of focus were excluded from the analysis. Thus, manual correction of plotting errors of automated segmentation was not performed in this study.

Methodology for pRNFL imaging in Cirrus and Spectralis has been reported previously [3]. Briefly, cross-sectional imaging of the peripapillary area was performed using Cirrus OCT. pRNFL thickness was determined using the optic disc cube protocol (software version 5.1.1.6) that generates a cube of data through a $6 \mathrm{~mm}$ square grid. A $3.46 \mathrm{~mm}$ in diameter calculation circle was automatically positioned around the optic disc. Cirrus OCT provides average pRNFL thickness and maps with 4 quadrants (superior, inferior, nasal, and temporal) and 12 clock hours, including classification from an internal normative database.

Spectralis OCT (software version 5.2.0.3) simultaneously captures infrared fundus and SD-OCT images at 40,000 Ascans per second. A real-time eye-tracking system measures eye movements and provides feedback to the scanning mechanism to stabilize the retinal position of the B-scan. The instrument uses 1024 A-scan points from a $3.45 \mathrm{~mm}$ circle centered on the optic disc. The examiner is required to manually place the scan around the optic disc. Peripapillary RNFL measurements were obtained using the N-site 


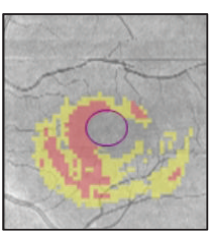

(a)

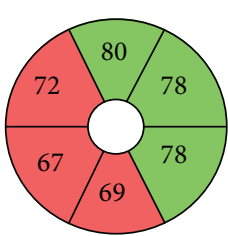

(b)

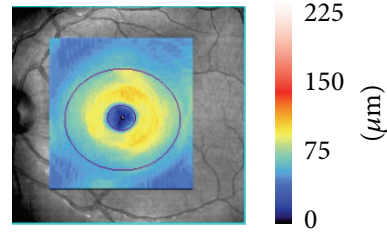

(c)

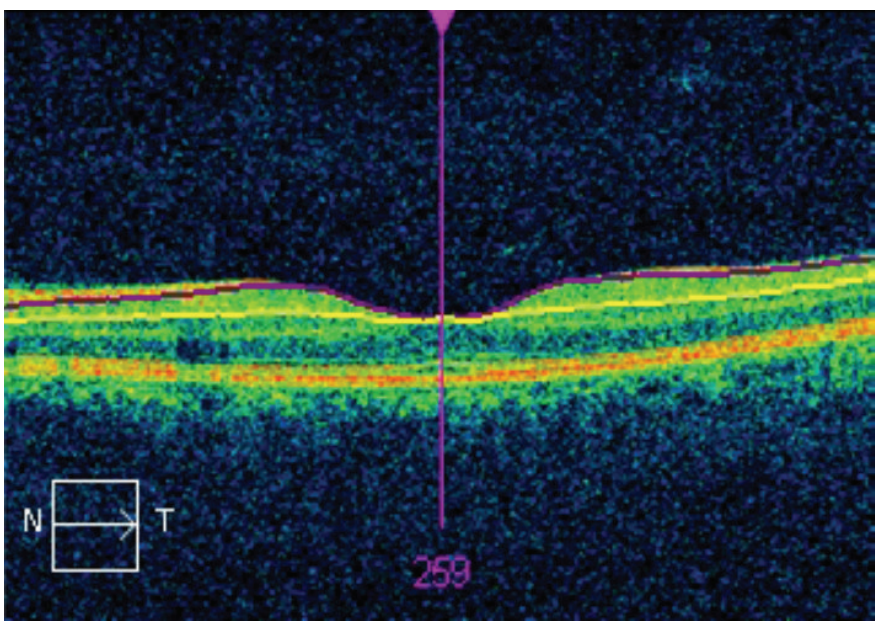

(d)

FIGURE 1: Ganglion cell-inner plexiform layer (GCIPL) analysis of the left eye of a patient with relapsing-remitting multiple sclerosis without optic neuritis antecedent in a $6 \times 6 \times 2 \mathrm{~mm}$ macular cube using a Cirrus optical coherence tomography. (a) Deviation map of the GCIPL thickness (red: below percentile 1; yellow: below percentile 5). (b) Sector distribution. (c) GCIPL thickness map, with overlying ellipses showing the dimensions of the analyzed annulus. The outer ellipse has a vertical diameter of $4 \mathrm{~mm}$ and the inner ellipse of $1 \mathrm{~mm}$. (d) Horizontal B-scan centered in the fovea, showing the automated segmentation of the GCIPL.

axonal protocol, which differs from the standard pRNFL scan because it starts and terminates in the nasal side of optic nerve. Scans were obtained using the high resolution (HR) mode and using automatic real-time (ART) for averaging 9 B-scan frames in order to improve image quality. The pRNFL Spectralis protocol generates a map showing the average thickness, maps with 4 quadrants (superior, inferior, nasal, and temporal), and maps with 6 sector thicknesses (superonasal, nasal, inferonasal, inferotemporal, temporal, and superotemporal).

The pRNFL thicknesses in the normal range are represented by green backgrounds. Those that are abnormal at the $5 \%$ and at the $1 \%$ level are represented by yellow and red backgrounds, respectively. The hypernormal (95th to 100th percentiles) pRNFL thicknesses are presented by a white color in Cirrus and by a blue/purple color in Spectralis.

Cross-sectional imaging of the macular area was performed using Cirrus OCT macular cube $(512 \times 128)$. This acquisition protocol generates a cube through a $6 \mathrm{~mm}$ square grid of 128 B-scans of 512 A-scans each. A built-in GCIPL analysis algorithm detects and measures the thickness of the macular GCIPL within a $6 \times 6 \times 2 \mathrm{~mm}$ elliptical annulus area centered on the fovea. The annulus has an inner vertical diameter of $1 \mathrm{~mm}$, which was chosen to exclude the portions of the fovea where the layers are very thin and difficult to detect accurately, and an outer vertical diameter of $4 \mathrm{~mm}$, which was chosen according to where the GCL again becomes thin and difficult to detect. The GCA algorithm identifies the outer boundaries of the RNFL and IPL. The difference between the RNFL and the IPL outer boundary segmentations yields the combined thickness of the RGC layer and IPL. Cirrus OCT provides quantitative assessment of the ganglion cell and inner plexiform layers (GCIPL) in 6 circular sectors centered in the fovea (superonasal, superior, inferonasal, inferotemporal, inferior, and superotemporal). It also gives information on the mean and minimum GCIPL thickness for each eye and compares these figures with a normative database (Figure 1). The GCIPL thicknesses in the normal range are represented by green backgrounds. Those that are abnormal at the $5 \%$ and at the $1 \%$ level are represented by yellow and red backgrounds, respectively. The hypernormal (95th to 100th percentiles) pRNFL thicknesses are presented by a white color.

2.3. Statistical Analysis. Data were analyzed using Stata/SE 12.0 for Unix and IBM SPSS Version 20 for Unix. A $P$ value of less than 0.05 was considered statistically significant.

Quantitative variables were summarized as mean \pm standard deviation. Qualitative variables were summarized as absolute value (percentage). Generalized estimating equation models accounting for sex, age, and within-patient intereye correlations were used to examine correlations and associations between variables. 
When evaluating new medical diagnostic tests, data may be obtained from one or more tests, but none of these can be considered a gold standard, that is, a diagnostic test with $100 \%$ sensitivity and specificity [13]. Latent class analysis (LCA) is based on the concept that observed results of different imperfect tests for the same disease are influenced by a latent common variable, the true disease status, which cannot be directly measured. In a group of patients with unknown disease status, for whom results from several diagnostic tests are available, LCA will model the probability of each combination of test results on the latent class and will provide an estimate of sensitivity and specificity for each of the diagnostic tests evaluated $[14,15]$. LCA has been used extensively for the estimation of sensitivity and specificity of diagnostic tests in the absence of a valid gold standard, mainly in microbiology $[16,17]$ and psychology [18], but also in ophthalmology [19].

In this study, we implemented the basic latent class model, using the assumption of conditional independence given the latent class. In basic LCA, there are no associations between the observed variables within each category of the latent variable. The latent variable is the true status on the disease, and the hypothesis is that there are two latent classes (presence or absence of retinal thickness changes). As more than one pRNFL measure could not be fitted into the same model due to the conditional independence assumption, two LCA models were built. Four variables were included in each LCA; average and minimum macular GCIPL thicknesses by Cirrus OCT and BCVA were present in both models; temporal pRNFL thicknesses by Cirrus OCT or by Spectralis OCT were present in one model each. LCA requires tests with binary outcomes to create the model. For simplification of the analysis, white, blue, purple, and green sectors have been labeled as "normal," and yellow and red ones as "abnormal." For BCVA, values better than or equal to 0.3 LogMAR were labeled as normal and those worse than 0.3 as "abnormal." BCVA was included in the model in order to provide a functional outcome that could help better define the latent class "retinal thickness change." Temporal pRNFL was selected as it was the quadrant with a higher frequency of pRNFL thinning and abnormal results in previous studies [4, 20-23].

LCA was performed using TAGS software implemented in $\mathrm{R}$ version 2.2 ( $\mathrm{R}$ Development Core Team and R Foundation for Statistical Computing, 2005). The fit of LCA model for the assumption of conditional independence was performed through the goodness-of-fit test followed by the evaluation of residual correlations between tests.

\section{Results}

Seventy RRMS patients and seventy age- and gender-matched healthy controls were enrolled in the study. All participants were of Caucasian descent. Table 1 summarizes the demographic and clinical characteristics of the participants.

Overall, average pRNFL and temporal quadrant pRNFL thickness by both Cirrus and Spectralis OCTs were significantly lower in both $\mathrm{ON}$ and non-ON RRMS eyes compared to healthy eyes $(P<0.001)$. Similarly, average, minimum, and each of the 6 sectors GCIPL thicknesses yielded by Cirrus were significantly lower in RRMS compared to healthy eyes in both $\mathrm{ON}$ and non-ON eyes $(P<0.001)$.

All these measurements were significantly lower in eyes with a prior history of ON compared to non-ON eyes $(P<$ $0.001)$.

Table 2 shows the percentage of abnormal color-coded measurements (defined as red or yellow color codes) obtained by GCIPL and pRNFL analysis in healthy and RRMS patients. Abnormal results were significantly more common in $\mathrm{ON}$ and non-ON RRMS eyes versus healthy eyes and in eyes with ON antecedent versus those without this antecedent in RRMS patients.

Overall, the highest abnormal percentage was observed in minimum (47.8\%) followed by average (46.4\%) GCIPL analysis. The sector in GCIPL test showing the highest abnormality rate was the superonasal (47.1\%) followed by superotemporal sector $(45.7 \%)$. The abnormality rates were significantly higher in eyes with a prior ON compared to nonON eyes (Figure 2 ).

Using Cirrus OCT, average GCIPL was altered more frequently than average pRNFL in eyes of patients with RRMS (46\% versus 33\%, resp.; $P<0.001$ ).

In a subgroup analysis comparing abnormal results between GCIPL and pRNFL by ON antecedent, average and minimum GCIPL measurements yielded the highest abnormal results for both $\mathrm{ON}$ and non-ON eyes (Table 2).

Table 3 shows the estimated sensitivity and specificity to detect retinal thickness changes by OCT with the two LCA models. The test for evaluating the fit of the model with conditional independence (goodness-of-fit test) proved to be adjusted $(P$ value $=0.938$ for model $\mathrm{A}$ and 0.836 for model B). The residual correlations between tests were randomly distributed around 0 .

Both GCIPL measurements appeared to be more sensitive and specific than temporal pRNFL thickness measured by Cirrus or Spectralis OCT (Table 3). Estimated sensitivities using Cirrus were 96.34\%, 98.43\%, and 58.41\% for average, minimum GCIPL, and temporal pRNFL, respectively. Using Spectralis, estimated sensitivity for temporal pRNFL was lower $(69.69 \%)$ than for Cirrus GCIPL measurements (average: $97.15 \%$ and minimum: $96.41 \%$ ).

Importantly, average GCIPL thickness was significantly more sensitive than temporal pRNFL by Cirrus $(P<0.05)$, and minimum GCIPL was significantly more sensitive than temporal pRNFL by Spectralis for the detection of retinal thickness changes in RRMS $(P<0.05)$. The model appeared to be robust, as sensitivities and specificities for both GCIPL measurements and BCVA were similar in both models.

Abnormal results in average GCIPL thickness in RRMS patients were independently associated to age in years $(\mathrm{OR}=$ $0.942, P=0.030)$, years since the diagnosis of RRMS (OR = $1.185, P=0.002)$, and $\mathrm{ON}$ antecedent $(\mathrm{OR}=4.123, P=$ $0.001)$, after correcting by sex and intereye correlation using binary logistic generalized estimating equations $(N=140)$.

Generalised estimating equations accounting for sex, age, and within-patient intereye correlation were used to measure standardised correlations between pRNFL and GCIPL 


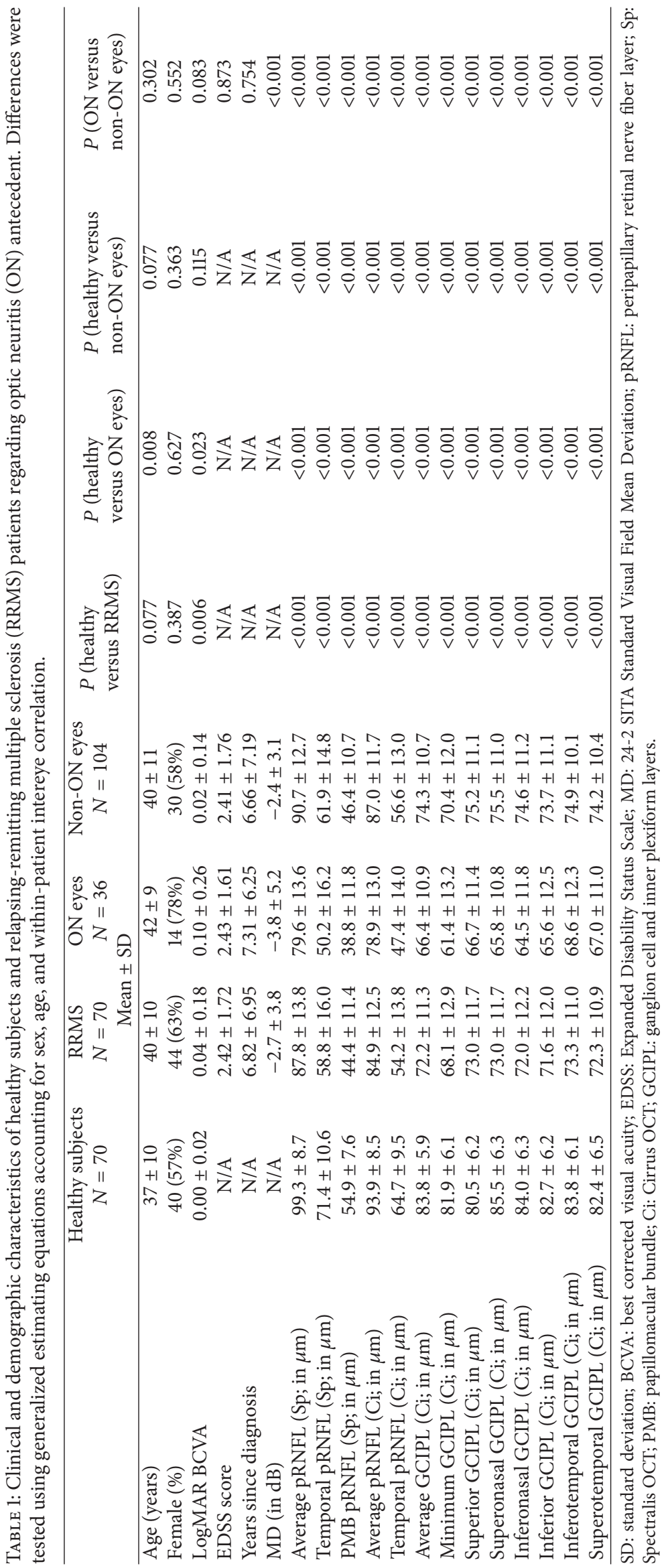




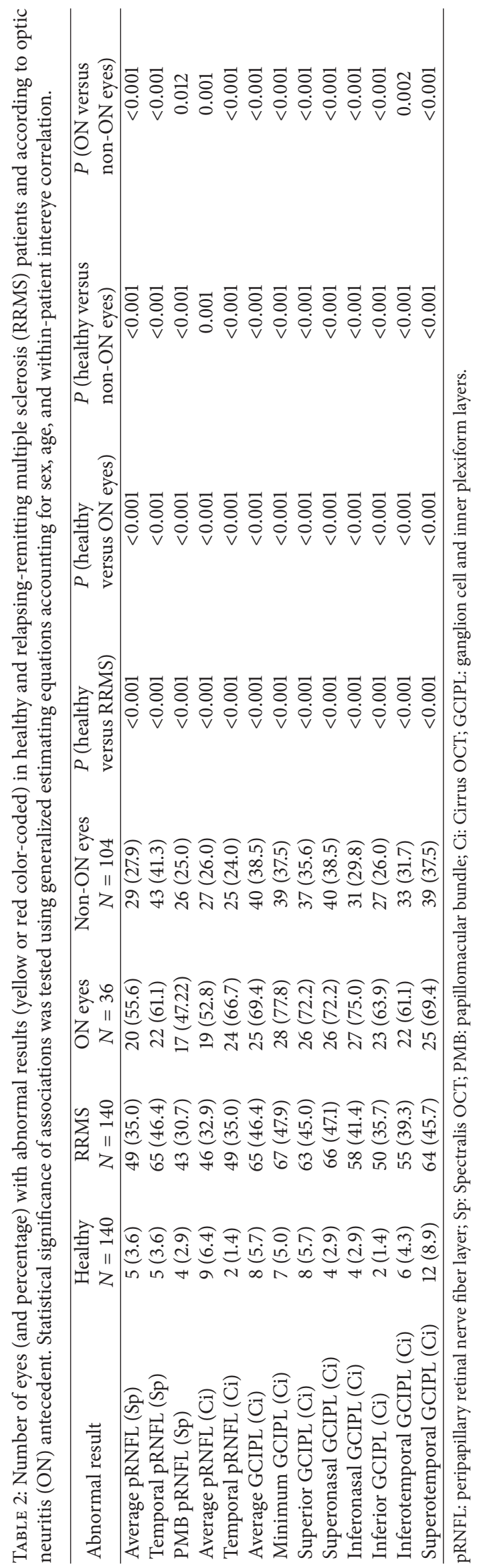




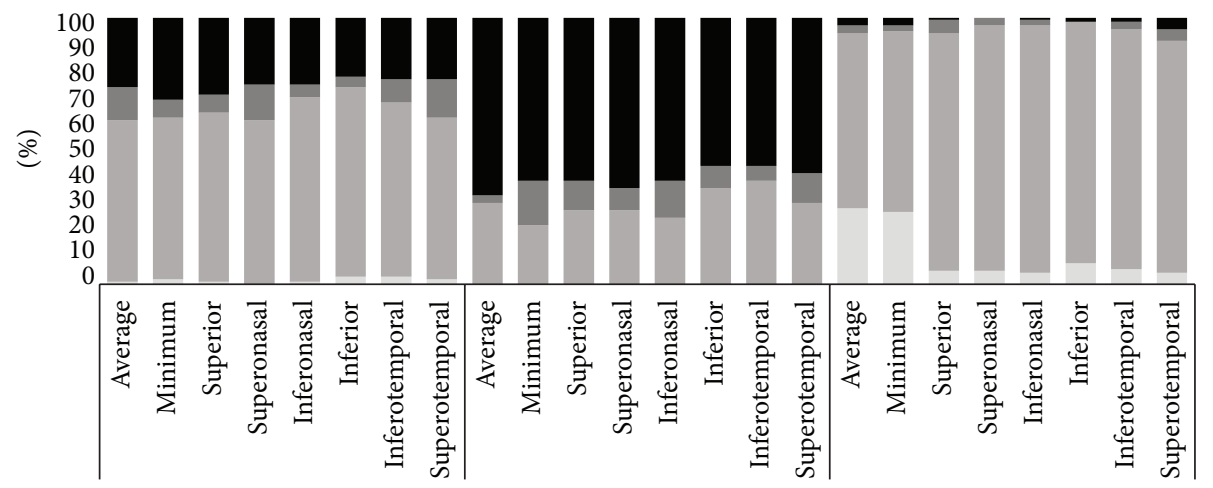

Eyes without optic neuritis

Eyes with optic neuritis

Control eyes

$$
\text { White } \quad \text { Yellow }
$$

FIGURE 2: Comparison of the color scale frequency for each sector using ganglion cell-inner plexiform layer analysis with Cirrus optical coherence tomography among eyes with and without optic neuritis in relapsing-remitting multiple sclerosis patients and from healthy control subjects. Black represents eyes classified as red (below percentile 1); dark gray represents eyes labeled as yellow (below percentile 5); light gray represents green (between percentiles 5 and 95), and white represents white (above percentile 95).

thicknesses and disease duration, EDSS, and visual function parameters (Table 4).

Average pRNFL thickness measured with Cirrus $(\beta=$ $-0.233 ; P=0.031)$ and Spectralis $(\beta=-0.228 ; P=0.025)$ OCTs, temporal $(\beta=-0.261 ; P=0.017)$ and papillomacular bundle $(\beta=-0.275 ; P=0.006)$ pRNFL thickness measured with Spectralis OCT, average GCIPL thickness $(\beta=-0.262$; $P=0.026)$, and minimum GCIPL thickness $(\beta=-0.299$; $P=0.008)$ correlated inversely with disease duration.

A strong, positive correlation was observed between average GCIPL thickness and pRNFL thickness using Cirrus $(\beta=0.692 ; P<0.001)$ and Spectralis $(\beta=0.642 ; P<0.001)$ OCTs.

After adjusting by age, sex, and within-patient intereye correlation, Cirrus GCIPL average and minimum measures showed a weak but significant correlation with EDSS only in eyes with ON antecedent (Table 4).

\section{Discussion}

Loss of RNFL is a well-documented structural marker of axonal degeneration in the eyes of patients with multiple sclerosis with and without a history of ON [8, 24-26]. Historically, OCT studies in multiple sclerosis have focused mostly on the RNFL, but retinal ganglion cell neuronal loss may also be implicated in the pathogenesis of visual dysfunction in MS [10].

To the best of our knowledge, apart from direct comparisons between pRNFL and GCIPL measurements in RRMS and healthy eyes [7-10], there is no information about the sensitivity and specificity of the different measurements to detect retinal thickness changes in RRMS patients.

Although strong positive correlation was found between GCIPL and pRNFL thickness values, average and minimum GCIPL measures showed higher sensitivity and specificity than temporal pRNFL measures. Remarkably, average
GCIPL showed a significantly better sensitivity than temporal pRNFL by Cirrus, and minimum GCIPL showed a significantly better sensitivity than temporal pRNFL by Spectralis for the detection of retinal thickness changes in RRMS.

In agreement with previous studies [21], we found that eyes from patients with RRMS had significant thinning in average and temporal quadrant pRNFL values by Cirrus and Spectralis OCT, and, in each of six sectors, average and minimum GCIPL results obtained by Cirrus, when compared to healthy eyes (Table 1). This was true for eyes with and without $\mathrm{ON}$ antecedent. Nevertheless, eyes with $\mathrm{ON}$ history showed greater $\mathrm{PRNFL}$ and GCIPL thinning than eyes without $\mathrm{ON}$ antecedent [10] (Table 1).

In a previous study [4] analyzing the color-code classification of pRNFL in RRMS patients, we identified the temporal quadrant to be the most abnormally color-coded by both Cirrus and Spectralis. Additionally, temporal pRNFL quadrants were abnormally color-coded more frequently in ON eyes than in non-ON eyes by both devices, Cirrus and Spectralis.

The abnormality rate in temporal pRNFL color code in eyes with previous history of $\mathrm{ON}$ was $66.7 \%$ by Cirrus and $61.1 \%$ by Spectralis. The current study agrees with previous reports that eyes from patients with RRMS exhibit a significant thinning of the pRNFL and GCIPL compared with the healthy eyes $[7,10,11]$ and that pRNFL thinning in RRMS patients typically occurs in the temporal sector $[4,20-$ 23, 27-29].

As expected, the sector showing the highest abnormality rate in GCIPL test was the superonasal (47.1\%).

Unsurprisingly, GCIPL thinning showed an association with disease duration and $\mathrm{ON}$ antecedent in eyes of patients with RRMS. GCIPL minimum represents the lowest GCIPL thickness over a single meridian crossing the annulus, which is expected to be sensitive to focal damage [30]. This measurement had previously been found to have the highest correlation with visual field pattern standard deviation in patients 
TABLE 3: Estimated sensitivity and specificity of different optic coherence tomography measures for retinal thickness changes detection in eyes of patients with relapsing-remitting multiple sclerosis $(N=140)$ and from healthy control subjects $(N=140)$ using latent class analysis. Model A: temporal pRNFL thickness as measured by Cirrus OCT; estimated prevalence of retinal thickness changes in the sample was $23.42 \%$; 95\% confidence interval (95CI) 18.50 to $29.17 \%$. Model B: temporal pRNFL thickness as measured by Spectralis OCT; estimated prevalence of retinal thickness changes in the sample was $24.25 \%$; $95 \%$ confidence interval (95CI) 19.26 to $30.04 \%$.

\begin{tabular}{|c|c|c|c|c|}
\hline & \multicolumn{2}{|c|}{ Sensitivity } & \multicolumn{2}{|c|}{ Specificity } \\
\hline & Estimate & $95 \mathrm{CI}$ & Estimate & 95CI \\
\hline \multicolumn{5}{|l|}{ Model A } \\
\hline Cirrus average GCIPL & $96.34 \%$ & 76.11 to $99.54 \%$ & $97.05 \%$ & 92.77 to $98.83 \%$ \\
\hline Cirrus minimum GCIPL & $98.43 \%$ & 64.61 to $99.95 \%$ & $95.81 \%$ & 91.00 to $98.10 \%$ \\
\hline Cirrus temporal pRNFL & $58.41 \%$ & 45.82 to $70.00 \%$ & $93.91 \%$ & 89.56 to $96.52 \%$ \\
\hline Best corrected visual acuity & $6.14 \%$ & 2.32 to $15.30 \%$ & $98.59 \%$ & 95.72 to $99.54 \%$ \\
\hline \multicolumn{5}{|l|}{ Model B } \\
\hline Cirrus average GCIPL & $97.15 \%$ & 76.94 to $99.71 \%$ & $97.61 \%$ & 93.16 to $99.19 \%$ \\
\hline Cirrus minimum GCIPL & $96.41 \%$ & 81.48 to $99.39 \%$ & $95.43 \%$ & 90.80 to $97.78 \%$ \\
\hline Spectralis temporal pRNFL & $69.69 \%$ & 57.14 to $79.86 \%$ & $92.70 \%$ & 88.01 to $95.65 \%$ \\
\hline Best corrected visual acuity & $6.08 \%$ & 2.30 to $15.15 \%$ & $98.55 \%$ & 95.58 to $99.53 \%$ \\
\hline
\end{tabular}

GCIPL: ganglion cell-inner plexiform layers; pRNFL: peripapillary retinal nerve fiber layer.

TABLE 4: Standardized correlation coefficients between OCT measurements and neurologic and visual function parameters in eyes of patients with relapsing-remitting multiple sclerosis, calculated using generalized estimating equations accounting for sex, age, and within-patient intereye correlation $(n=140)$.

\begin{tabular}{|c|c|c|c|c|}
\hline & BCVA & $\mathrm{MD}$ & EDSS & Disease duration \\
\hline Cirrus average pRNFL & $0.286^{*}$ & $0.418^{*}$ & -0.014 & $-0.233^{\ddagger}$ \\
\hline Cirrus temporal pRNFL & -0.013 & 0.062 & 0.001 & -0.141 \\
\hline Spectralis average $\mathrm{pRNFL}$ & $0.314^{*}$ & $0.360^{*}$ & 0.033 & $-0.228^{\ddagger}$ \\
\hline Spectralis temporal pRNFL & $0.122^{\ddagger}$ & $0.268^{\dagger}$ & -0.101 & $-0.261^{\ddagger}$ \\
\hline Spectralis PMB pRNFL & $0.125^{\ddagger}$ & $0.209^{\ddagger}$ & -0.066 & $-0.275^{\dagger}$ \\
\hline GCIPL average & $0.226^{*}$ & $0.513^{*}$ & -0.178 & $-0.262^{\ddagger}$ \\
\hline GCIPL minimum & $0.204^{\dagger}$ & $0.412^{*}$ & -0.161 & $-0.299^{\dagger}$ \\
\hline \multicolumn{5}{|c|}{ Non-ON eyes $(N=104)$} \\
\hline Cirrus average pRNFL & -0.019 & 0.098 & 0.033 & $-0.253^{\ddagger}$ \\
\hline Cirrus temporal pRNFL & $-0.270^{\ddagger}$ & -0.030 & -0.046 & -0.146 \\
\hline Spectralis average $\mathrm{pRNFL}$ & $0.210^{*}$ & 0.145 & 0.109 & $-0.274^{\ddagger}$ \\
\hline Spectralis temporal pRNFL & -0.025 & 0.093 & -0.092 & $-0.284^{\dagger}$ \\
\hline Spectralis PMB pRNFL & 0.002 & 0.083 & -0.040 & $-0.332^{\dagger}$ \\
\hline GCIPL average & -0.062 & 0.109 & -0.113 & $-0.297^{\ddagger}$ \\
\hline GCIPL minimum & 0.029 & 0.138 & -0.092 & $-0.314^{\dagger}$ \\
\hline \multicolumn{5}{|c|}{ ON eyes $(N=36)$} \\
\hline Cirrus average pRNFL & 0.188 & 0.161 & -0.173 & 0.012 \\
\hline Cirrus temporal pRNFL & -0.043 & 0.001 & 0.105 & -0.013 \\
\hline Spectralis average $\mathrm{pRNFL}$ & 0.175 & 0.149 & -0.151 & 0.109 \\
\hline Spectralis temporal pRNFL & 0.112 & 0.030 & -0.195 & 0.065 \\
\hline Spectralis PMB pRNFL & 0.059 & -0.022 & -0.120 & 0.097 \\
\hline GCIPL average & 0.145 & 0.133 & $-0.429^{\dagger}$ & -0.079 \\
\hline GCIPL minimum & 0.144 & 0.120 & $-0.421^{\ddagger}$ & -0.227 \\
\hline
\end{tabular}

${ }^{*} P<0.001 ;{ }^{\dagger} P<0.01 ;{ }^{\ddagger} P<0.05$.

OCT: optic coherence tomography; BCVA: best corrected visual acuity; MD: Goldman 24-2 SITA standard visual field mean deviation; EDSS: Expanded Disability Status Scale; pRNFL: peripapillary retinal nerve fiber layer; PMB: papillomacular bundle; GCIPL: ganglion cell and inner plexiform layers; ON: optic neuritis. 
with chronic open angle glaucoma [31]. In our study, Cirrus GCIPL minimum measures showed a significant correlation with BCVA, mean deviation, and EDSS. Importantly, only GCIPL measures showed significant correlation with EDSS in ON eyes (Table 4).

A superior structure-function correlation between GCIPL thickness and clinical measures compared to pRNFL has been reported recently, suggesting that GCIPL analysis might be a better approach than pRNFL to examine MS neurodegeneration [11].

Previous studies have shown that GCIPL thickness can be altered in patients with RRMS [10, 32-34] and that these alterations correlated with visual function $[10,11]$ and central nervous system findings using magnetic resonance imaging [28]. Some of these studies have suggested that GCIPL thickness can be a more reliable measure for the detection of retinal anomalies in RRMS than pRNFL thickness [10]. Interestingly, our study demonstrates that a decrease in GCIPL thickness is more sensitive to retinal involvement in RRMS than an alteration in the temporal pRNFL.

This study has a number of limitations warranting discussion. Firstly, exact sensitivity and specificity cannot be obtained without a gold standard test. The values obtained through LCA can be useful when comparing different tests; however, the sensitivities and specificities provided are estimates. Studies with bigger sample size and including information on other diagnostic tests, such as electrodiagnostic testing and magnetic resonance imaging, could help to improve this estimation. Secondly, both eyes were included in this study. However, most MS studies published to date have included both eyes because they can be individually evaluated and do not necessarily follow the same disease course $[1,4]$. Additionally, generalized estimating equations were used in order to account for sex, age, and within-patient intereye correlation. With these methods, information from both eyes can be used for the study, without the risk of increasing the risk of bias due to intereye correlation or increase in sample size [35].

The retinal segmentation algorithm used by Cirrus OCT combines GCL and IPL, since the boundaries between these two layers cannot be visually discriminated on this device. Although Spectralis OCT has developed a specific software that provides automated differentiation and quantification of the individual retinal layers, it was not available when the data was collected. Additionally, this software does not provide comparison to a normative database, so binary outcomes necessary for LCA would not be available.

Finally, we have included only patients with relapsingremitting multiple sclerosis; therefore, our results cannot be extrapolated to other types of multiple sclerosis or to patients with more advanced disease (mean EDSS was 2.42).

\section{Conclusions}

In conclusion, OCT GCIPL analysis is more sensitive than temporal pRNFL analysis to detect retinal thickness changes in RRMS eyes. GCIPL measures correlate better than pRNFL measures with visual function parameters such as BCVA, visual field mean deviation, or EDSS. As such, GCIPL thickness measured by retinal segmentation of OCT scans may be an ideal marker for monitoring neurodegeneration in RRMS patients.

\section{Conflict of Interests}

The authors have no proprietary or commercial interest in any materials discussed in this paper. All authors have completed and submitted the ICMJE form for disclosure of potential conflict of interests and none were reported.

\section{Authors' Contribution}

Conception and design was done by Julio J. GonzálezLópez, Gema Rebolleda, Francisco J. Muñoz-Negrete, Marina Leal, Noelia Oblanca, Lucienne Costa-Frossard, and José C. Álvarez-Cermeño; analysis and interpretation was done by Julio J. González-López and Gema Rebolleda; writing the paper was done by Julio J. González-López, Gema Rebolleda, and Francisco J. Muñoz-Negrete; critical revision of the paper was done by Marina Leal, Noelia Oblanca, Lucienne Costa-Frossard, and José C. Álvarez-Cermeño. Final approval of the paper was done by Julio J. González-López, Gema Rebolleda, Francisco J. Muñoz-Negrete, Marina Leal, Noelia Oblanca, Lucienne Costa-Frossard, and José C. ÁlvarezCermeño; data collection was done by Julio J. GonzálezLópez, Marina Leal, and Noelia Oblanca; provision of materials, patients, or resources was done by Gema Rebolleda, Francisco J. Muñoz-Negrete, Lucienne Costa-Frossard, and José C. Álvarez-Cermeño; statistical expertise was provided by Julio J. González-López; obtaining funding was done by Gema Rebolleda and Francisco J. Muñoz-Negrete; and literature search was done by Julio J. González-López and Gema Rebolleda.

\section{Acknowledgments}

Julio J. González-López is a Ph.D. candidate at the Surgery Department, Universidad de Alcalá School of Medicine, Madrid, Spain. This study has been granted by the "Fondo de Investigaciones Sanitarias," from the Spanish Ministry of Health and Consumer Affairs.

\section{References}

[1] E. Ojeda, D. Díaz-Cortes, D. Rosales, C. Duarte-Rey, J.-M. Anaya, and A. Rojas-Villarraga, "Prevalence and clinical features of multiple sclerosis in Latin America," Clinical Neurology and Neurosurgery, vol. 115, no. 4, pp. 381-387, 2013.

[2] F. Ikuta and H. M. Zimmerman, "Distribution of plaques in seventy autopsy cases of multiple sclerosis in the United States," Neurology, vol. 26, part 2, no. 6, pp. 26-28, 1976.

[3] R. H. Kardon, "Role of the macular optical coherence tomography scan in neuro-ophthalmology," Journal of NeuroOphthalmology, vol. 31, no. 4, pp. 353-361, 2011.

[4] G. Rebolleda, J. J. González-López, F. J. Muñoz-Negrete, N. Oblanca, L. Costa-Frossard, and J. C. Álvarez-Cermeño, "Colorcode agreement among stratus, cirrus, and spectralis optical coherence tomography in relapsing-remitting multiple sclerosis 
with and without prior optic neuritis," The American Journal of Ophthalmology, vol. 155, no. 5, pp. 890-897, 2013.

[5] A. J. Green, S. McQuaid, S. L. Hauser, I. V. Allen, and R. Lyness, "Ocular pathology in multiple sclerosis: retinal atrophy and inflammation irrespective of disease duration," Brain, vol. 133, part 6, pp. 1591-1601, 2010.

[6] K. S. Shindler, E. Ventura, M. Dutt, and A. Rostami, "Inflammatory demyelination induces axonal injury and retinal ganglion cell apoptosis in experimental optic neuritis," Experimental Eye Research, vol. 87, no. 3, pp. 208-213, 2008.

[7] P. Albrecht, M. Ringelstein, A. K. Müller et al., "Degeneration of retinal layers in multiple sclerosis subtypes quantified by optical coherence tomography," Multiple Sclerosis, vol. 18, no. 10, pp. 1422-1429, 2012.

[8] S. B. Syc, S. Saidha, S. D. Newsome et al., "Optical coherence tomography segmentation reveals ganglion cell layer pathology after optic neuritis," Brain, vol. 135, part 2, pp. 521-533, 2012.

[9] L. S. Talman, E. R. Bisker, D. J. Sackel et al., "Longitudinal study of vision and retinal nerve fiber layer thickness in multiple sclerosis," Annals of Neurology, vol. 67, no. 6, pp. 749-760, 2010.

[10] S. D. Walter, H. Ishikawa, K. M. Galetta et al., "Ganglion cell loss in relation to visual disability in multiple sclerosis," Ophthalmology, vol. 119, no. 6, pp. 1250-1257, 2012.

[11] S. Saidha, S. B. Syc, M. K. Durbin et al., "Visual dysfunction in multiple sclerosis correlates better with optical coherence tomography derived estimates of macular ganglion cell layer thickness than peripapillary retinal nerve fiber layer thickness," Multiple Sclerosis, vol. 17, no. 12, pp. 1449-1463, 2011.

[12] C. H. Polman, S. C. Reingold, G. Edan et al., "Diagnostic criteria for multiple sclerosis: 2005 revisions to the 'McDonald Criteria," Annals of Neurology, vol. 58, no. 6, pp. 840-846, 2005.

[13] M. Sadatsafavi, N. Shahidi, F. Marra et al., "A statistical method was used for the meta-analysis of tests for latent TB in the absence of a gold standard, combining random-effect and latent-class methods to estimate test accuracy," Journal of Clinical Epidemiology, vol. 63, no. 3, pp. 257-269, 2010.

[14] S. L. Hui and S. D. Walter, "Estimating the error rates of diagnostic tests," Biometrics, vol. 36, no. 1, pp. 167-171, 1980.

[15] D. Rindskopf and W. Rindskopf, "The value of latent class analysis in medical diagnosis," Statistics in Medicine, vol. 5, no. 1, pp. 21-27, 1986.

[16] E. Girardi, C. Angeletti, V. Puro et al., "Estimating diagnostic accuracy of tests for latent tuberculosis infection without a gold standard among healthcare workers," Euro Surveillance, vol. 14, no. 43, 2009.

[17] T. S. Machado de Assis, A. Rabello, and G. L. Werneck, "Latent class analysis of diagnostic tests for visceral leishmaniasis in Brazil," Tropical Medicine and International Health, vol. 17, no. 10, pp. 1202-1207, 2012.

[18] M. Shevlin, S. Murphy, and J. Murphy, "Adolescent loneliness and psychiatric morbidity in the general population: Identifying "at risk" groups using latent class analysis," Nordic Journal of Psychiatry, 2014.

[19] M. Ang, W. L. Wong, X. Li, and S.-P. Chee, "Interferon $\gamma$ release assay for the diagnosis of uveitis associated with tuberculosis: a Bayesian evaluation in the absence of a gold standard," The British Journal of Ophthalmology, vol. 97, no. 8, pp. 1062-1067, 2013.

[20] C. Fjeldstad, M. Bemben, and G. Pardo, "Reduced retinal nerve fiber layer and macular thickness in patients with multiple sclerosis with no history of optic neuritis identified by the use of spectral domain high-definition optical coherence tomography," Journal of Clinical Neuroscience, vol. 18, no. 11, pp. 14691472, 2011.

[21] E. Garcia-Martin, V. Pueyo, I. Pinilla, J.-R. Ara, J. Martin, and J. Fernandez, "Fourier-domain OCT in multiple sclerosis patients: reproducibility and ability to detect retinal nerve fiber layer atrophy," Investigative Ophthalmology and Visual Science, vol. 52, no. 7, pp. 4124-4131, 2011.

[22] S. Noval, I. Contreras, S. Munoz, C. Oreja-Guevara, B. Manzano, and G. Rebolleda, "Optical coherence tomography in multiple sclerosis and neuromyelitis optica: an update," Multiple Sclerosis International, vol. 2011, Article ID 472790, 11 pages, 2011.

[23] G. Rebolleda, A. García-García, H. R. Won Kim, and F. J. MũozNegrete, "Comparison of retinal nerve fiber layer measured by time domain and spectral domain optical coherence tomography in optic neuritis," Eye, vol. 25, no. 2, pp. 233-238, 2011.

[24] K. M. Galetta, P. A. Calabresi, E. M. Frohman, and L. J. Balcer, "Optical coherence tomography (OCT): imaging the visual pathway as a model for neurodegeneration," Neurotherapeutics, vol. 8, no. 1, pp. 117-132, 2011.

[25] A. Petzold, J. F. de Boer, S. Schippling et al., "Optical coherence tomography in multiple sclerosis: a systematic review and metaanalysis," The Lancet Neurology, vol. 9, no. 9, pp. 921-932, 2010.

[26] S. Saidha, S. B. Syc, M. A. Ibrahim et al., "Primary retinal pathology in multiple sclerosis as detected by optical coherence tomography," Brain, vol. 134, part 2, pp. 518-533, 2011.

[27] J. B. Fisher, D. A. Jacobs, C. E. Markowitz et al., "Relation of visual function to retinal nerve fiber layer thickness in multiple sclerosis," Ophthalmology, vol. 113, no. 2, pp. 324-332, 2006.

[28] E. García-Martín, V. Pueyo, J. Martin et al., "Progressive changes in the retinal nerve fiber layer in patients with multiple sclerosis," European Journal of Ophthalmology, vol. 20, no. 1, pp. 167-173, 2010.

[29] V. Pueyo, J. R. Ara, C. Almarcegui et al., "Sub-clinical atrophy of the retinal nerve fibre layer in multiple sclerosis," Acta Ophthalmologica, vol. 88, no. 7, pp. 748-752, 2010.

[30] J.-C. Mwanza, M. K. Durbin, D. L. Budenz et al., "Profile and predictors of normal ganglion cell-inner plexiform layer thickness measured with frequency-domain optical coherence tomography," Investigative Ophthalmology \& Visual Science, vol. 52, no. 11, pp. 7872-7879, 2011.

[31] H. El Chehab, M. Delbarre, J. Fenolland et al., "Structure function relationship: specific index of ganglion cell complex assessment of 2 spectral domain OCT and standard automated perimetry," Acta Ophthalmologica Scandinavica, vol. 91, supplement s252, 2013.

[32] D. B. Fernandes, A. S. Raza, R. G. Nogueira et al., "Evaluation of inner retinal layers in patients with multiple sclerosis or neuromyelitis optica using optical coherence tomography," Ophthalmology, vol. 120, no. 2, pp. 387-394, 2013.

[33] J. N. Ratchford, S. Saidha, E. S. Sotirchos et al., "Active MS is associated with accelerated retinal ganglion cell/inner plexiform layer thinning," Neurology, vol. 80, no. 1, pp. 47-54, 2013.

[34] S. Saidha, E. S. Sotirchos, J. Oh et al., "Relationships between retinal axonal and neuronal measures and global central nervous system pathology in multiple sclerosis," Archives of Neurology, vol. 70, no. 1, pp. 34-43, 2013.

[35] I. E. Murdoch, S. S. Morris, and S. N. Cousens, "People and eyes: statistical approaches in ophthalmology," British Journal of Ophthalmology, vol. 82, no. 8, pp. 971-973, 1998. 


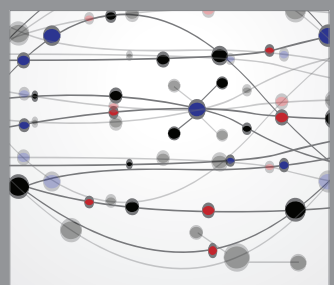

The Scientific World Journal
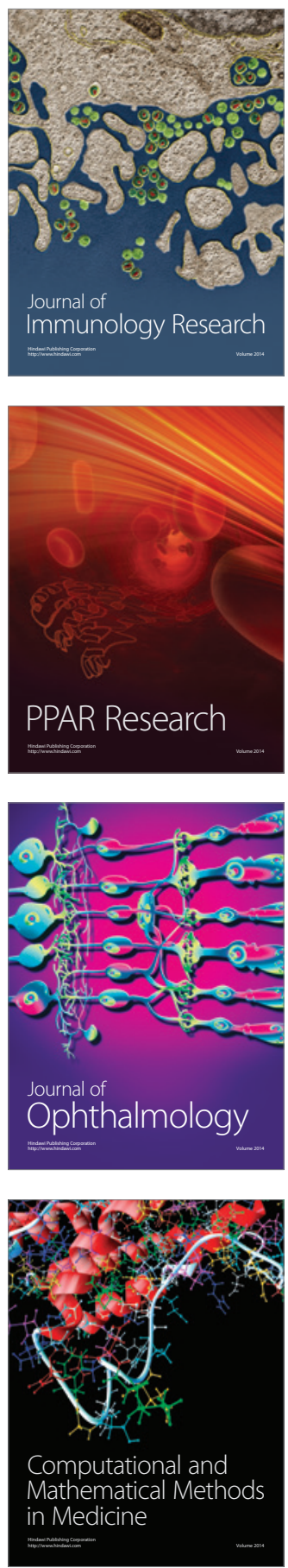

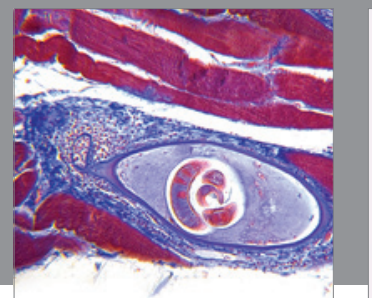

Gastroenterology

Research and Practice
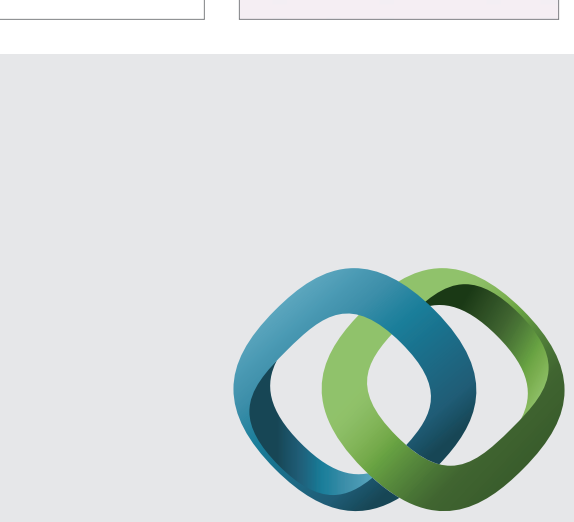

\section{Hindawi}

Submit your manuscripts at

http://www.hindawi.com
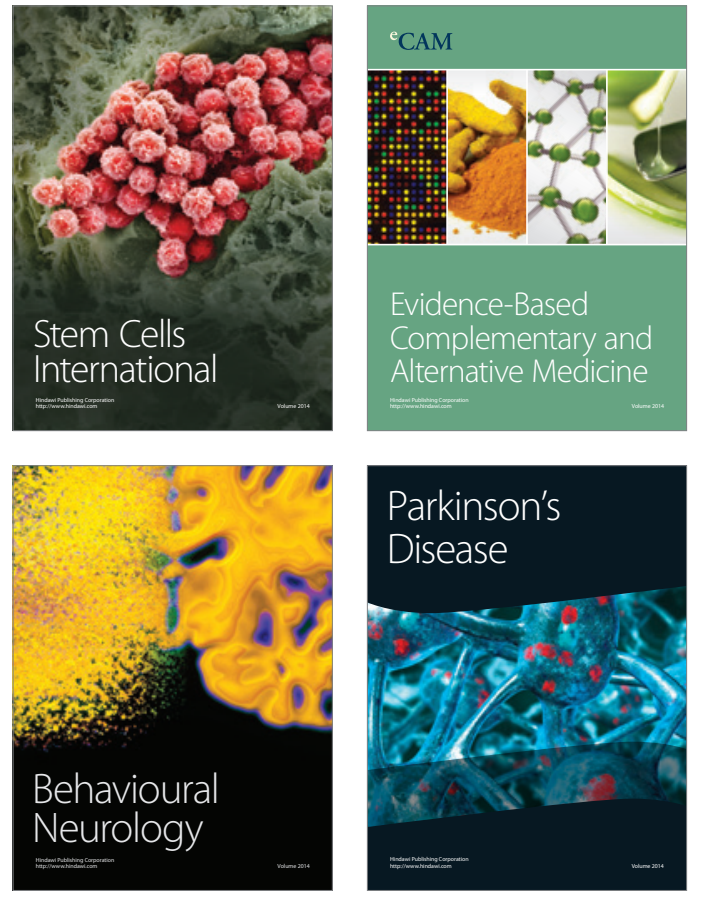
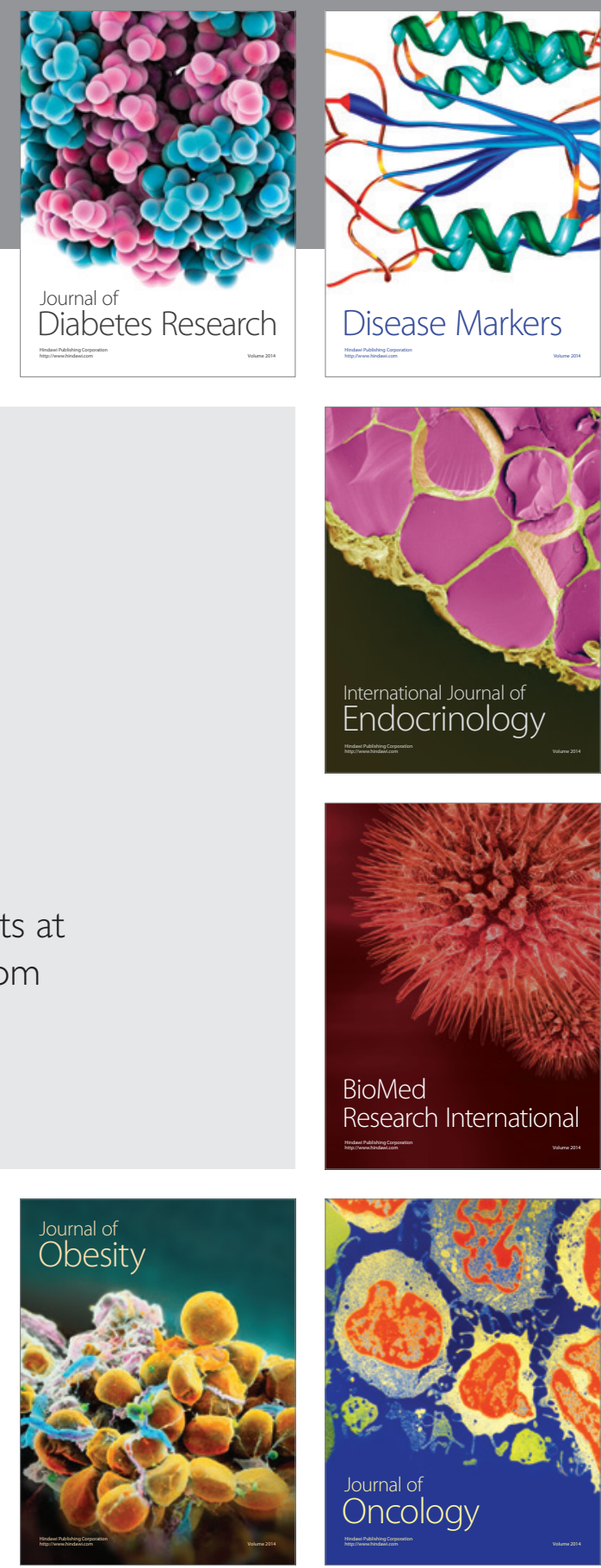

Disease Markers
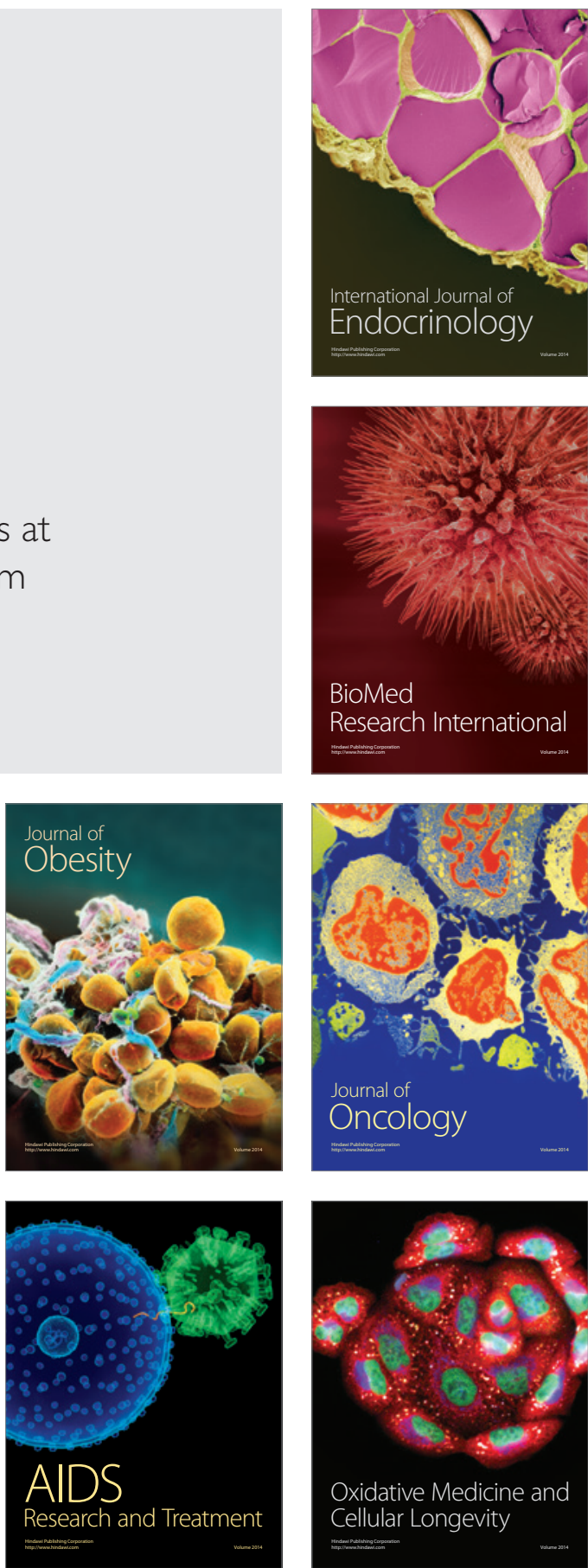